\title{
Suivis multifréquences, caractérisation des stocks sédimentaires et modélisation morpho-dynamique d'un environnement littoral mixte
}

\author{
Alexandre NICOLAE LERMA ${ }^{1}$, Damien DAILLOUX ${ }^{2}$, François PARIS ${ }^{1}$ \\ 1. BRGM (French Geological Survey), Regional Direction Nouvelle-Aquitaine, 24 \\ Avenue Léonard de Vinci, 33600 Pessac, France. \\ a.nicolaelerma@brgm.fr \\ 2. CASAGEC Ingénierie, 18 Rue Maryse Bastié, 64600 Anglet, France. \\ dailloux@casagec.fr
}

\section{Résumé :}

La connaissance des stocks de sédiments disponibles à l'échelle spatiale de l'ensemble d'un système côtier (des plages aux zones subtidales) et la compréhension des dynamiques de transit sont fondamentales pour mener des actions de gestion du littoral. Dans un contexte composé de falaises, de récifs subtidaux et de plages de poches, les stocks de sable accumulés sur les plages peuvent connaître des évolutions rapides et complexes. Le site d'étude est situé au niveau de la commune de Bidart, sur la côte Basque française. Plus particulièrement, la plage du Centre est emblématique de ces dynamiques. Le site connaît une trajectoire d'évolution pluriannuelle d'érosion du haut de plage, contraignant les gestionnaires à envisager un réaménagement et une réhabilitation du front de mer. Cette étude propose, à partir de la compilation de plusieurs jeux de données et de différents outils d'observation (levés topo-bathymétriques, levés sismiques, carottages de sédiments, LiDAR aérien et suivi vidéo), une analyse des évolutions morphologiques et des stocks sédimentaires disponibles en zones subtidales et intertidales aux échelles de temps interannuelles, saisonnières et hebdomadaires. Ce suivi multifréquence et l'évaluation de la position du socle rocheux ainsi que la variabilité de la quantité et de la position des stocks sableux sont mis à profit pour la mise en place et le calage d'un modèle d'évolution morpho-dynamique (X-Beach). Ainsi, des scénarios de forçage et l'analyse des impacts en termes d'érosion en fonction des stocks sédimentaires disponibles sont réalisés. Ce modèle constitue un outil déterminant pour l'étude des solutions d'aménagement envisageables sur ce tronçon littoral.

Mots-clés : Plage de poche, Stocks sédimentaires, Renaturation du front de mer, X-Beach, Côte Basque.

\section{Introduction}

En général, peu d'études ont été menées sur les échanges de sédiments entre les petits fonds et les plages dans des environnements mixtes (rocheux/sableux) soumis à des conditions de forte énergie (MUÑOZ-PEREZ \& MEDINA, 2010 ; GALLOP et al., 


\section{Thème 2 - Dynamique sédimentaire}

2012). Les stocks de sable peuvent connaitre des évolutions rapides et complexes à interpréter car les transites sédimentaires résultent à la fois de forte contraintes induites par le contexte géologique et des dynamiques fluctuantes dans le temps, en fonction de la marée, de l'énergie saisonnière des vagues et de leurs directions de provenance.

Sur le littoral de la commune de Bidart, et particulièrement de la plage du Centre la disponibilité des stocks de sédiments sableux et leurs dynamiques sont mal connues. Les importantes variations ses dernières années ont conduit à la déstabilisation des aménagements en haut de plage et à l'étude de projets de renaturation du front de mer.

Cette étude propose, à partir de la compilation de plusieurs jeux de données et de différents outils d'observation (levés topo-bathymétriques, levés sismiques, carottages de sédiments, LiDAR aérien et suivi vidéo), une analyse des évolutions morphologiques et des stocks sédimentaires disponibles en zones subtidales et intertidales aux échelles de temps interannuelles, saisonnières et hebdomadaires. Les connaissances acquises sont mises à profit pour la validation et la réalisation de scénarios d'évolution morphodynamiques contraintes par la nature mixte de l'environnement littoral (contrainte géologique). Une discussion est menée sur le fonctionnement global de la plage du centre et la nécessité de prendre en compte ces évolutions à différentes échelles de temps pour la mise en place d'actions de gestion ou de renaturation du haut de plage.

\section{Site d'étude}

Le site d'étude est localisé sur la commune de Bidart sur la côte basque française (figure 1). Le littoral y est caractérisé par une succession de falaises, de plateformes rocheuses et de secteurs d'accumulation constituant des plages de poches par endroits perchées sur une dalle rocheuse. Les sédiments sont constitués de sables fins à moyens dont la granulométrie est relativement uniforme (D50 entre 0,5 et 0,05 mm). Les marées sont semi-diurnes d'amplitude méso à macrotidale (marnage moyen de 3,75 m). Sur toute la période étudiée (janvier 2008 à mai 2019), les vagues incidentes proviennent presque exclusivement du secteur Ouest-Nord-Ouest à Nord-Ouest, entre 300 et $315^{\circ} \mathrm{N}$ (figure 1). On observe une légère différence de direction entre les saisons automne-hiver où le mode directionnel se situe autour de 300 à $330^{\circ} \mathrm{N}$ et les saisons printemps-été où le mode se situe plutôt autour de $290-300^{\circ} \mathrm{N}$. Du point de vue de l'intensité, les plus fortes houles (Hs supérieures à $3 \mathrm{~m}$ ) peuvent être observées d'octobre à avril et dépassent fréquemment 5 $\mathrm{m}$ de novembre à février. 


\section{XVİ̀mes Journées Nationales Génie Côtier - Génie Civil \\ Le Havre 2020}
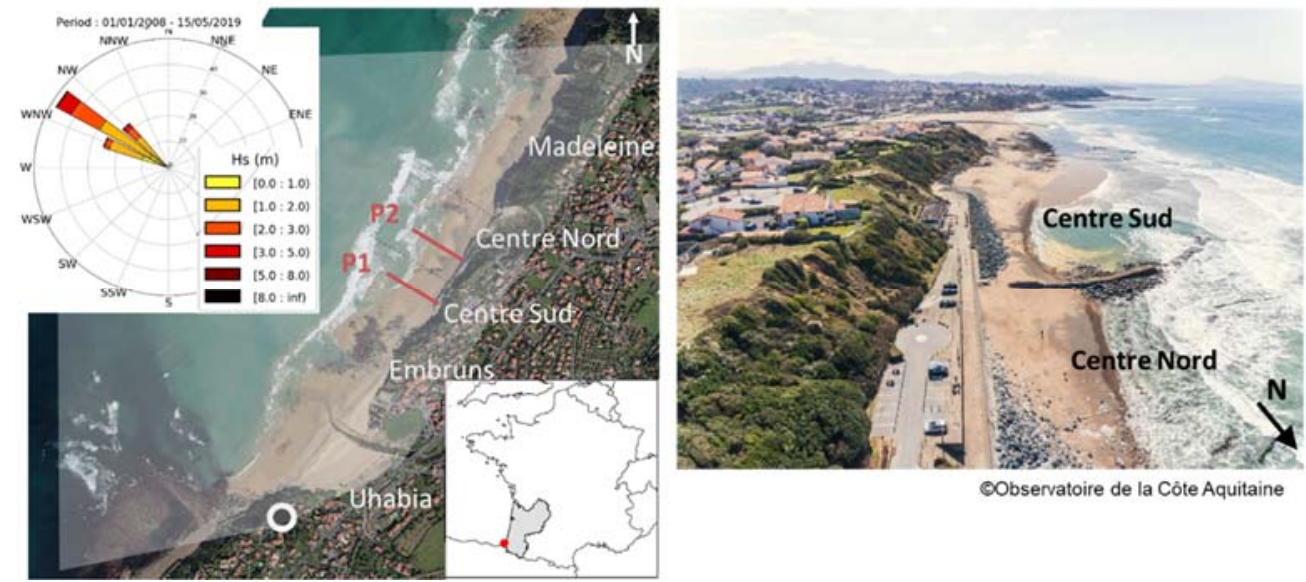

Figure 1. Carte de localisation et rose des houles (données MARC (www.umrlops.org/marc, période 2008-2019). Le cercle blanc et la zone grisée représentent respectivement la localisation de la station vidéo et son emprise.

\section{Données et méthode}

Ce travail repose sur des données acquises dans le cadre du projet MAREA (Programme européen POCTEFA 2017-2019, NICOLAE LERMA et al., 2019) et des données plus anciennes collectées par l'OCA (Observatoire de la Côte Aquitaine) et CASAGEC ingénierie. Les données couvrent la période 2009-2019 avec des fréquences d'acquisition variables (voir tableau 1).

Les évolutions des stocks et des morphologies associées sont restituées à des fréquences hebdomadaires, mensuelles, saisonnières et interannuelles à l'aide de plusieurs campagnes de terrain couvrant une distance long-shore d'environ 2,3 km (figure 1) et s'étendant du haut de plage jusqu'aux profondeurs avoisinant les -20 $\mathrm{m}$ : (i) 7 campagnes aériennes de LiDAR topographiques et ortophotographiques, (ii) 17 levés D-GPS à pied ou sur quad, (iii) 6 levés bathymétriques.

Tableau 1. Synthèse des acquisitions de données.

\begin{tabular}{llll}
\hline Outils & Période d'acquisition & Fréquence d'acquisition & Nombre de d'acquisition \\
\hline D-GPS & $2009-2016$ & Annuelle & 8 \\
& $2017-2019$ & Trimestrielle à mensuelle & 9 \\
LiDAR & 2011 & - & 7 \\
& $2014-2019$ & Annuelle & \\
Sondeur bathymétrique & $2009-2011$ & Annuelle & 3 \\
& $2017-2019$ & Biannuelle & 4 \\
Vidéo & $2017-2019$ & Continue & - \\
Sondage géologique & 2017 & - & 1 \\
Sonar & 2017 & - & 1 \\
Sondeur sédiment & 2018 & - & 1 \\
\hline
\end{tabular}




\section{Thème 2 - Dynamique sédimentaire}

Dans le cadre de l'étude, CASAGEC a mis à disposition un système de suivi vidéo. Les données vidéo sont calibrées de manière à obtenir une mesure de l'altimétrie des niveaux de sable en pied d'ouvrage (figure 2). La calibration a consisté en la mesure simultanée de (i) l'altimétrie de plusieurs points de référence localisés sur plusieurs transects verticaux répartis le long de l'ouvrage, (ii) et la digitalisation de ces points sur l'image vidéo. Cette technique permet d'obtenir la relation entre la taille des pixels au niveau des profils et le nivellement réel avec une précision de l'ordre de 15 à $20 \mathrm{~cm}$, ici au niveau de l'épi de la plage de Bidart Centre.

Les données vidéo ont été traitées entre Novembre 2017 et Février 2019 afin d'obtenir au minimum une série de 4 images par mois à marée basse.
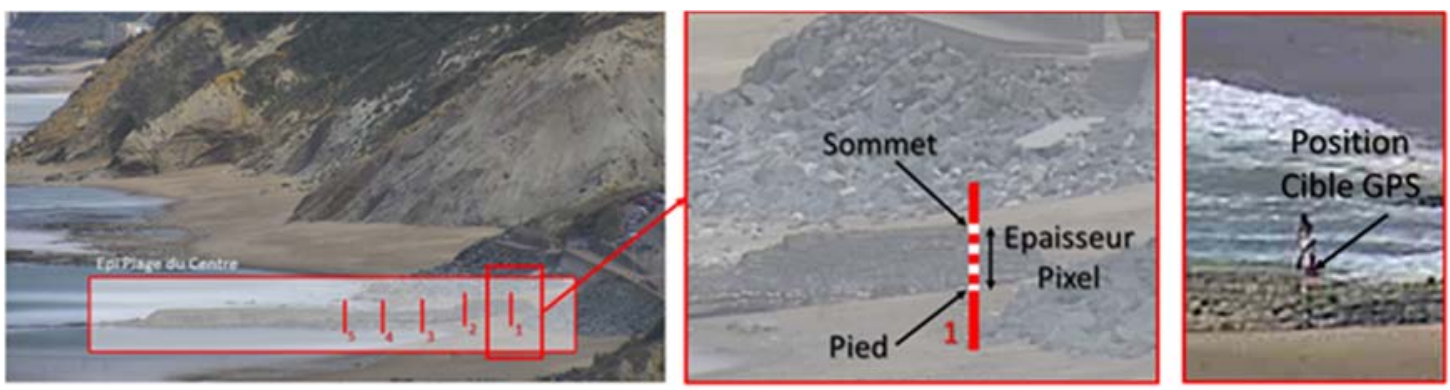

Figure 2. Digitalisation de cible GPS au niveau de l'épi de la Centrale, et zoom sur la digitalisation d'un point GPS.

Associé à ces acquisitions de surface, des campagnes géophysiques ont été réalisées afin de déterminer la position du toit rocheux en zone intertidale et subtidale (environ $20 \mathrm{~m}$ de profondeur). Ainsi, l'évaluation de l'épaisseur des sédiments a été effectuée en utilisant (i) une campagne de 50 forages géologiques sur les plages intertidales et supratidales, (ii) un levé au sonar à balayage latéral de type Tritech Starfish 452F afin de déterminer les faciès sédimentaires, (iii) un levé au sondeur à sédiment de type Pinger SBP permettant de déterminer la position du substrat rocheux pour la partie marine. La disponibilité des stocks de sédiments ou USU (Upper Sand Unit) est ainsi évaluée par la différence entre la profondeur du substratum rocheux et la surface topo-bathymétrique.

\section{Résultats}

\subsection{Evolutions morphologiques et échelles temporelles}

Les évolutions morphologiques des plages et la quantité de sédiments mobilisés peuvent être très importantes sur des échelles de temps court (à l'échelle de temps d'une marée lors de conditions agitées). Les épaisseurs moyennes de sédiments à l'échelle saisonnière varient fortement, de l'ordre de 0 à $3 \mathrm{~m}$ entre les plages et $10 \mathrm{~m}$ de profondeur. La figure 3 présente des différentiels d'évolution caractéristiques à différentes échelles spatiotemporelles. 


\section{XVİ̀mes Journées Nationales Génie Côtier - Génie Civil \\ Le Havre 2020}

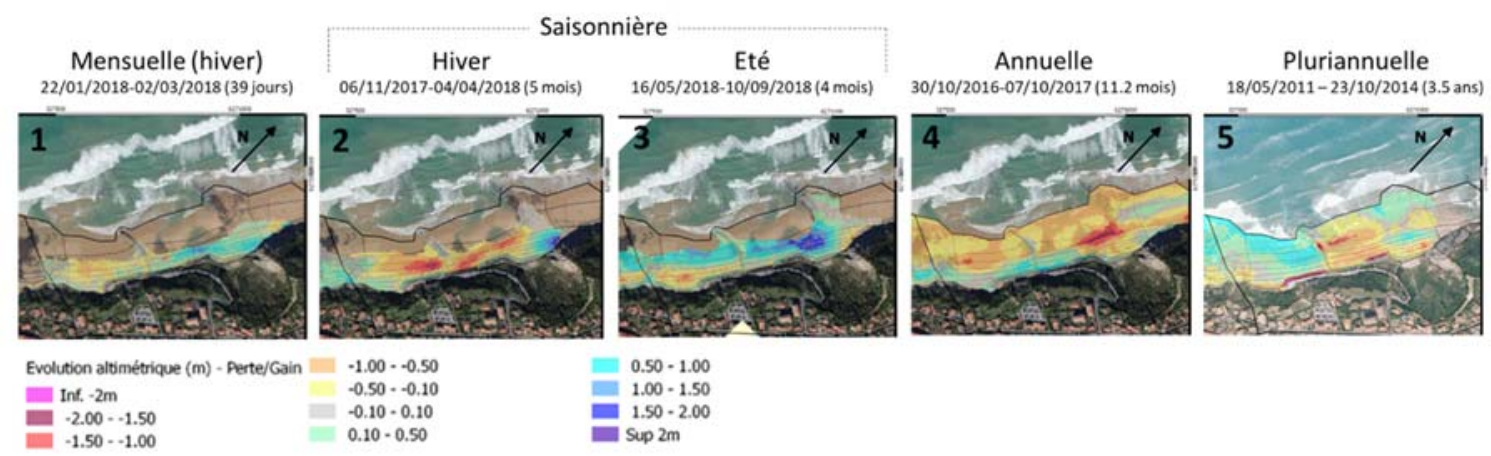

Figure 3. Exemple d'évolutions multi-temporelles de la plage du Centre.

Les transferts de sable sont principalement caractérisés par de mouvements transversaux (cross-shore) qui traduisent la mise en équilibre du système plage en fonction de l'énergie des houles, des niveaux d'eau et des stocks de sable disponibles et qui induisent des transferts depuis les petits fonds vers la plage ou inversement. Des transferts "longshore" peuvent également être observés et conduire à des accumulations de part et d'autre de l'épi central. La figure 3 illustre :

(1) Un comportement différent des plages Nord et Sud à échelle mensuelle suggérant des transferts à dominante long-shore Sud-Nord ( + ou $-1 \mathrm{~m}$ à $1,5 \mathrm{~m})$;

(2) et (3) Des bascules cross-shore saisonnières de transferts de sable entre les petitsfonds et les plages. $(+$ ou $-1,5$ à $2 \mathrm{~m})$;

(4) Une diminution de la quantité de sédiments présents sur les plages en situation préhivernale entre 2016 et 2017 (de 0,5 à $2 \mathrm{~m}$ ) ayant conduit à la déstabilisation des aménagements de haut de plage lors de l'hiver 2017-2018.

(5) Une très nette différence d'évolution des plages Sud et Nord à échelle de temps pluriannuelle. Ces évolutions, qui se caractérisent par des mouvements très contrastés des stocks sableux entre la plage Sud et Nord, sont détaillées dans la partie suivante.

\subsection{Trajectoires d'évolutions contrastées}

Les évolutions des volumes des stocks de sédiments disponibles aux dates d'acquisition sont présentées à la figure 4. En 2009, on estime un volume total de $61000 \mathrm{~m}^{3}$ de sable présent sur les plages du Centre, avec une répartition de $35000 \mathrm{~m}^{3}$ sur la plage Centre Nord et de $26000 \mathrm{~m}^{3}$ sur la plage Centre Sud. En 2019, la cubature fait état d'une très forte diminution du stock global estimé à $25000 \mathrm{~m}^{3}$. Alors que le volume pour la plage du Sud est stable sur la période 2009-2019, le volume sur la plage Nord a diminué de plus de $70 \%$. Au total le système de la plage du Centre (Sud et Nord confondu) a perdu plus de la moitié de son volume de sable sur 10 ans (-41\%). Deux points principaux caractérisent le fonctionnement de ce système sur la période 2009-2019 :

a) Une très grande variabilité saisonnière avec des engraissements ou des érosions massives et rapides. Dernièrement, le système gagne plus de $30 \%$ pendant l'été 2018 et perd $40 \%$ pendant l'hiver 2018-2019. 


\section{Thème 2 - Dynamique sédimentaire}

b) La plage Nord et la plage Sud suivent des dynamiques différentes sur la période étudiée. Les stocks de sable diminuent fortement sur la plage Nord alors que la plage Sud est stable.
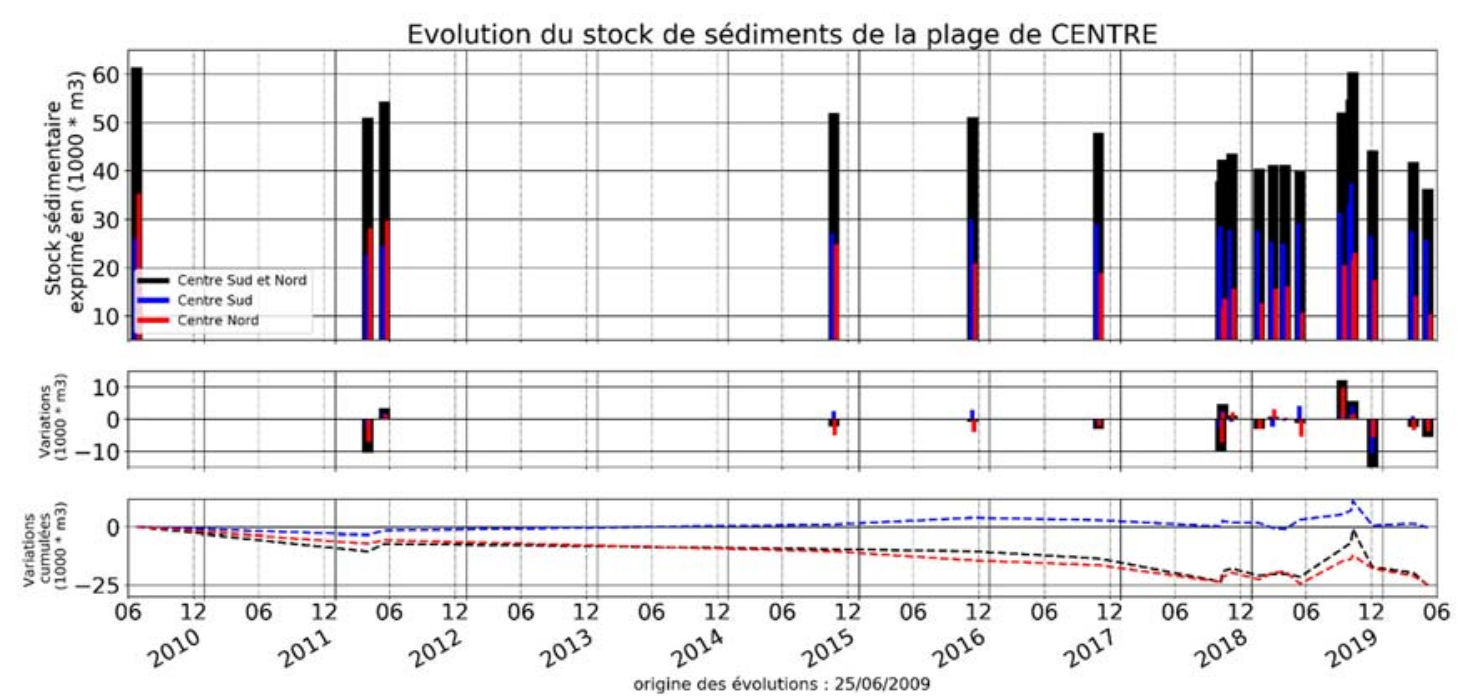

Figure 4. Evolution des stocks sédimentaires sur les plages du Centre de 2009 à 2019.

4.3 Evolution haute fréquence et modélisation morpho-dynamique évènementielle L'analyse des données vidéo montre que les fluctuations de niveau de sable augmentent de l'enracinement de l'épi vers son musoir. De l'ordre de $1 \mathrm{~m}$ à $1,5 \mathrm{~m}$ aux profils 1 à 3 , elles atteignent environ $2 \mathrm{~m}$ aux profils 4 et 5 . Les niveaux les plus bas sont atteints au cours du printemps 2018, entre les mois de Mars et de Mai.

Les données topo-bathymétriques et les données géophysiques ont permis la mise en place en configuration 1D du modèle Xbeach (ROELVINK et al., 2009) au niveau d'un profil cross-shore localisé sur la plage de Bidart Centre (figure 5 et 6). Parallèlement, les données du modèle ont été confrontées aux résultats de suivi vidéo haute fréquence pour validation. Ainsi, l'utilisation du modèle a permis d'améliorer les connaissances sur l'impact morpho sédimentaire des tempêtes en fonction de différentes configurations de plage. 


\section{XVİ̀mes Journées Nationales Génie Côtier - Génie Civil \\ Le Havre 2020}

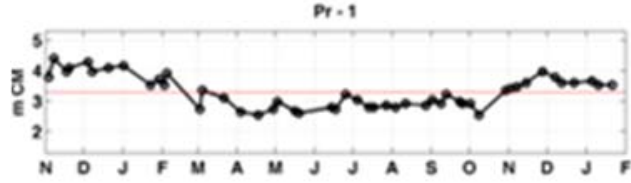

Pr:3

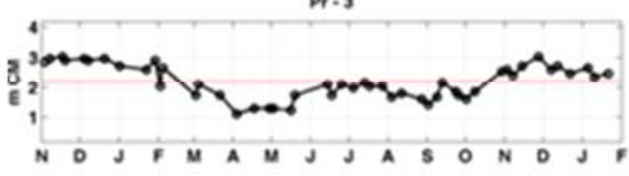

Pr-s

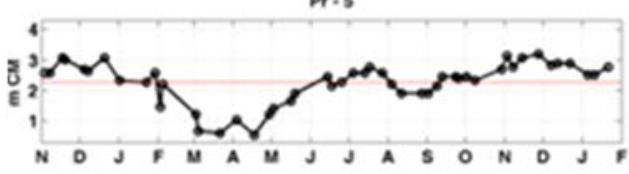

Pr.2

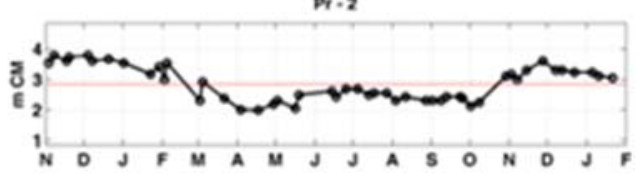

Pr: 4

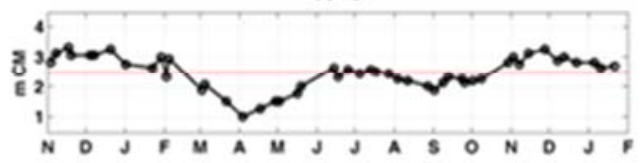

Figure 5. Résultats des évolutions topographiques au niveau l'épi de la Plage du Centre de Bidart entre Novembre 2017 et Février 2019.
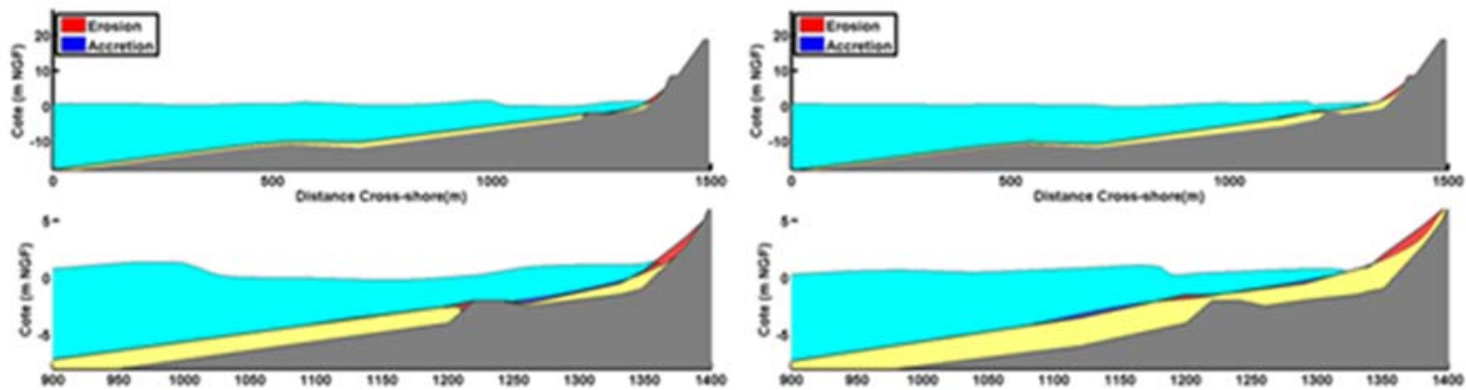

Figure 6. Exemple de modélisation Xbeach au niveau du profil P2, d'une tempête annuelle pour une configuration de faible ensablement (à gauche) et de fort ensablement (à droite). La partie inferieure des zooms au niveau de la plage.

\section{Discussion}

Les multiples outils et types d'acquisition permettent de retracer les évolutions de ce système de plage sur 10 ans avec des fréquences d'acquisitions augmentées pendant 3 ans afin d'évaluer la variabilité des morphologies et des stocks sédimentaires à haute fréquence (hebdomadaire, mensuelle et saisonnière).

L'analyse de ces évolutions à l'échelle de l'ensemble du système littoral de la commune de Bidart (de la plage de Parlementia à celle d'Erretegia) a permis de caractériser les grands modes de fonctionnement des systèmes plages (bascule transversale, rotation longitudinale) et de quantifier les mouvements sédimentaires (NICOLAE LERMA et al., 2020). Dans le cas spécifique de la plage du centre, la tendance observée est celle d'une réduction continue sur les 10 dernières années des stocks de sable en haut de plage au niveau de la plage du centre Nord. Cette tendance suggère que le système est en déséquilibre continu. Celui-ci étant potentiellement accentué par la présence de l'enrochement en haut de plage ancré relativement bas sur l'estran et en avancée par 


\section{Thème 2 - Dynamique sédimentaire}

rapport à la plage Sud. Toutefois, le fait de ne pas observer de tendance similaire sur la plage adjacente (centre Sud), la relativement forte disponibilité des sédiments dans la partie sous-marine et la capacité d'accumulation rapide de sédiment en haut de plage comme observé en automne 2018, laisse penser que le système pourrait se rééquilibrer moyennant une renaturation ou un réaménagement du haut de plage. Cette nouvelle configuration devra permettre des re-accumulations plus massives de sable en haut de plage potentiellement appuyées par des opérations de gestion.

\section{Conclusion}

La connaissance des stocks de sédiments disponibles à l'échelle spatiale de l'ensemble d'un système côtier (des plages aux zones subtidales) et la compréhension de la dynamique de transit au sein du système sont fondamentales pour les actions de gestion côtière. Le travail sur la caractérisation de l'état et de la dynamique des stocks sédimentaires des plages du Centre à Bidart a permis de mieux comprendre les évolutions aux échelles de temps évènementielles, saisonnières et pluriannuelle. L'ensemble des moyens de mesures déployés souligne le très grand dynamisme des stocks des petits fonds et des plages. Ces mesures ont également permis la mise en place de modélisations morpho-dynamiques contrainte grâce à l'identification du socle rocheux et des variations évènementielles observables sur ces plages. Elle constitue un préalable aux travaux de renaturation du littoral envisagés.

\section{Références bibliographiques}

GALlOP S.L., BOSSERELlE C., ELIOT I., PATTIARATCHI C.B. (2012). The influence of limestone reefs on storm erosion and recovery of a perched beach. Continental Shelf Research, Vol. 47, pp 16-27. https://doi.org/10.1016/j.csr.2012.08.001 MUÑOZ-PEREZ J.J., MEDINA R. (2010). Comparison of long-, medium- and shortterm variations of beach profiles with and without submerged geological control, Coastal Engineering, Vol. 57, pp 241-251. https://doi.org/10.1016/j.coastaleng.2009.09.011

NICOLAE LERMA A., PARIS F., VOIX F., GARNIER C. (2019). Caractérisation de l'état et de la dynamique des stocks sédimentaires des plages de Bidart et Guéthary en vue d'une gestion opérationnelle (Projet MAREA). Rapport final. BRGM/RP-69330-FR, $127 \mathrm{p}$.

NICOLAE LERMA A., PARIS F., GARCIN M., DAILLOUX D., (2020). Sandy stocks on a rocky shore: states and weekly to pluriannual evolutions. Journal of Coastal Research, Special Issue No. 95, pp 1-5.

ROELVINK D., RENIERS A., VAN DONGEREN A.P., DE VRIES J.V.T., MCCALL R., LESCINSKI J. (2009). Modelling storm impacts on beaches, dunes and barrier islands. Coastal Engineering, Vol. 56, pp 1133-1152. https://doi.org/10.1016/j.coastaleng.2009.08.006 